\title{
Análisis de la incidencia y duración de la formación laboral financiada por empresas y trabajadores*
}

\author{
Antonio Caparrós Ruiz \\ antonio@uma.es \\ M. ${ }^{a}$ Lucía Navarro Gómez \\ l_navarro@uma.es \\ Mario F. Rueda Narváez \\ mfrueda@uma.es \\ Departamento de Estadística y Econometría \\ Facultad de Ciencias Económicas \\ Universidad e Málaga \\ El Ejido s/n, 29071 Málaga
}

\section{RESUMEN}

Este trabajo pretende presentar evidencia empírica sobre los factores que determinan que empresas y trabajadores inviertan en formación laboral. Para ello, se utilizarán datos extraídos del PHOGUE (INE, 1995-2000). Desde el punto de vista econométrico, esto implica la estimación de modelos tobit de efectos aleatorios. Así, se estima no sólo la probabilidad de realizar algún curso de formación, sino también su duración. Por otra parte, el análisis también diferencia la formación según sea financiada por la empresa o los trabajadores. El considerar por separado el tipo de financiación se debe

* Este trabajo se ha realizado en el marco de los proyectos SEJ2007-68045-CO2-01/ECON del Ministerio de Educación y Ciencia y PRY116/09 del Centro de Estudios Andaluces. Asimismo, los autores agradecen los comentarios y sugerencias realizadas por dos evaluadores anónimos, que sin duda han contribuido a la mejora de este trabajo. 
a que, dependiendo de si los recursos los aportan la empresa o el asalariado, tanto la decisión como los costes y beneficios asociados recaerán en uno o en otro y, por tanto, es probable que la misma característica tenga un efecto diferente sobre los incentivos de los trabajadores y de los empresarios. Finalmente, el análisis se realizará por separado según género.

\begin{abstract}
This paper aims to present empirical evidence on the factors that affect how workers and employers decide to invest in training programs. To achieve this, we use data drawn from the Spanish section of the European Community Household Panel (ECHP; INE, 1995-2000). From the econometric point of view, our objective implies estimating tobit models for panel data with random effects. Thus, not only the probability of investing in training is estimated, but also the amount of training received, measured in days. The analysis is also carried out considering separately whether training is financed by the firm or the employee. We take this approach because it is reasonable to think that the costs and benefits of the decision will rest on whoever decides to invest and pay for these training courses. Therefore, it is possible that one variable has different effects on the incentives faced by workers and firms. Finally, the models are also estimated separately by gender.

Palabras clave: Capital humano, formación específica, modelos tobit con datos de panel.
\end{abstract}

Códigos JEL: C23, C24, J24.

\title{
1. INTRODUCCIÓN
}

La investigación en economía laboral durante las últimas décadas permite demostrar que el capital humano es un elemento clave a la hora de proporcionar a los trabajadores habilidades y, por lo tanto, productividad. En este sentido, la formación continua a lo largo de la vida profesional cumple un papel esencial para mantener actualizados los conocimientos de la fuerza de trabajo (Lynch, 1989), así como facilitar a los empleados el acceso a trayectorias laborales ascendentes (Blau y Khan, 1996). A la vista de su importancia, parece interesante conocer en detalle los mecanismos por los que empresas y trabajadores realizan este tipo de inversiones. Específicamente, cabe preguntarse de qué manera las ventajas de la formación se distribuyen entre la población 
asalariada. Es decir, si la formación beneficia a los que parten de una mejor posición (mayor nivel educativo, mejores empleos), amplificando las diferencias iniciales en el mercado de trabajo, o si por el contrario es un mecanismo que tiende a igualar las rentas de los trabajadores.

A partir de Becker (1964), se considera a la formación en el empleo como un tipo de capital humano, caracterizado fundamentalmente por adquirirse con posterioridad al abandono del sistema educativo y, en general, de forma simultánea al desarrollo de la actividad laboral. En cualquier caso, se trata de una inversión mediante la cual se sacrifican recursos a cambio de la esperanza de obtener en el futuro una mayor productividad que permita a su vez aumentar el salario.

El modelo original de Becker, basado en mercados de trabajo y de capital competitivos, dedica bastante atención a diferenciar la formación específica de la general. Mientras que esta última aumenta la productividad del trabajador en cualquier empresa en la que se encuentre, la formación específica sólo tiene efectos positivos en la propia empresa que la proporciona. A partir de esta distinción conceptual, se concluye que las empresas no invertirán en formación general, dado que los trabajadores podrían ponerla en uso en otra empresa, apropiándose de sus rendimientos. Por tanto, este tipo de formación sería costeada por los trabajadores. El argumento contrario sirve para las inversiones específicas. En este caso, los trabajadores no podrán amenazar a sus empleadores con abandonarlos a fin de conseguir un empleo en el que rentabilicen su formación, de modo que serían las empresas las encargadas de proporcionar la formación específica.

Con posterioridad, el modelo ha sido ampliado para tener en cuenta condiciones de competencia imperfecta e información asimétrica, lo que en cierta medida difumina la diferencia entre los dos tipos de formación considerados, dado que ciertas circunstancias pueden hacer que empresa y trabajador cofinancien la formación específica, compartiendo los rendimientos (Hashimoto, 1981), o bien pueden existir incentivos para que las empresas inviertan en formación general. Así, Acemoglu y Pischkle (1999) extienden el modelo de Becker para mostrar que si el incremento en productividad debido a la formación general no se traslada completamente a las ofertas salariales externas (por ejemplo, debido a que otras empresas no pueden conocer la calidad de la formación), entonces los empleadores invertirán en formación general. La razón se basa en que, aunque esta formación pueda ser teóricamente aprovechada en otras empresas, éstas no podrían distinguir a los trabajadores con más formación, por lo que sus ofertas salariales caerían por debajo de la verdadera productividad. Esto significa, en la práctica, convertir a la formación general en más o menos específica. Algo parecido ocurre si es la habilidad de los trabajadores lo que no es observado, generándose un problema de selección adversa. Chang y Wang (1995), partiendo del trabajo de Katz y Ziderman (1990), muestran cómo 
las empresas pueden utilizar la formación general para revelar la calidad de sus trabajadores. Después de recibir formación, los más hábiles no podrían aprovecharla en el exterior dado que los trabajadores menos hábiles son despedidos, por lo que las demás empresas, sin poder conocer la verdadera habilidad, tenderían a suponer que se trata de trabajadores despedidos y les ofrecerán salarios inferiores a su productividad ${ }^{1}$.

Los temas tratados en la investigación empírica sobre formación, por otra parte, no suelen ajustarse exactamente a la discusión teórica. Esto se debe a que los principales conceptos teóricos de interés (productividad, cantidades invertidas) son difíciles de medir y suelen estar ausentes en las encuestas habitualmente utilizadas en economía laboral, lo que limita las posibilidades y obliga a usar aproximaciones como salarios o variables de incidencia/duración de los cursos realizados. Esto es especialmente problemático en este campo: los salarios pueden separarse de la productividad cuando las empresas financian la formación, y ésta tiende a ser muy heterogénea, de modo que si sólo se conoce que un trabajador ha participado en actividades formativas sería difícil aproximar la cantidad invertida realmente. En general, las dos principales preocupaciones en el campo empírico son, de un lado, conocer la incidencia de la formación, con el objetivo de identificar a los grupos de trabajadores que más realizan este tipo de inversiones y, del otro, evaluar los efectos de éstas sobre los salarios.

La literatura disponible para España es relativamente escasa y reciente, dado que hasta hace pocos años no han aparecido fuentes estadísticas con la información necesaria. Un primer grupo de trabajos se ha centrado en los factores que determinan la inversión en formación formal (realización de cursos) o formación informal (learningby-doing), es decir, el tiempo necesario para que un trabajador desempeñe eficientemente sus tareas. Así, por un lado, a partir de investigaciones como García Espejo (1999) o Peraita (2000), es común encontrar que la probabilidad de recibir formación formal útil para el empleo es mayor para los trabajadores más jóvenes, con un nivel educativo más elevado y empleados en empresas grandes. Por otro lado, Caparrós y otros (2004) y Albert y otros (2005a) se interesan por un posible conflicto entre mayor flexibilidad laboral (que aumenta el número de contratos temporales) y formación formal, encontrando una relación negativa entre ambas. Finalmente, Salas-Velasco (2007) analiza los determinantes de la adquisición de formación informal en el empleo, para una muestra de individuos con estudios universitarios residentes en la provincia de Granada. Sus resultados más relevantes indican que los trabajadores infraeducados, los autoempleados y aquellos que desempeñan profesiones como abogado o médico son

${ }^{1}$ Una panorámica de las teorías sobre formación laboral puede encontrarse en Leuven (2005). 
los que necesitan una mayor adquisición de formación informal al inicio del empleo. A su vez, un segundo grupo de trabajos se ha centrado en los efectos de la formación sobre los salarios. Concretamente, Albert y otros (2005b) y Rueda (2006) verifican la existencia de un efecto positivo de la adquisición de capital humano útil para el empleo sobre las ganancias de los trabajadores.

En este contexto, el objetivo del presente trabajo es doble. En primer lugar, se trata de analizar los factores que llevan a empresas y trabajadores a invertir en formación a partir de los datos de asalariados españoles contenidos en el Panel de Hogares de la Unión Europea (INE, 1995-2000). En este caso, se realizan algunas ampliaciones al enfoque tradicional en las investigaciones empíricas. Así, se estudian por separado las muestras de hombres y mujeres, debido a las diferencias generales con las que ambos colectivos afrontan la vida laboral y, especialmente, con atención a las diferencias en responsabilidades familiares que pueden restringir la cantidad de recursos disponibles (especialmente en términos de tiempo) para invertir en formación. Además, aprovechando información contenida en la encuesta utilizada, se distingue entre la formación proporcionada por las empresas de la que no lo es. Mientras que el primer tipo de formación se supone financiada por las empresas, en el segundo caso se entiende que son los trabajadores los que la financian, ya sea directamente pagando algún tipo de derechos de matrícula para acceder a los cursos o, aún en caso de que los suministre gratuitamente alguna administración pública, soportando el coste de oportunidad correspondiente (pues la asistencia a estas actividades les quita tiempo que de otra manera podría haber sido utilizado en actividades laborales o de ocio). La distinción entre estos dos tipos de formación es importante, dado que permite tener en cuenta que los incentivos de la inversión sean distintos para los dos agentes. En segundo lugar, no sólo se estudia la probabilidad de recibir formación, sino también cuánto dura, ya que trabajar con una variable cuantitativa permitirá aproximar mejor la cantidad de recursos invertidos en la formación frente a la alternativa de un simple indicador binario de incidencia (trabajadores con/sin formación). Finalmente, se aprovecha la naturaleza longitudinal del PHOGUE para utilizar métodos de datos de panel que permitan tener en cuenta la existencia de heterogeneidad inobservable.

La organización del trabajo es la siguiente. En el segundo epígrafe se presentan los datos empleados y se detalla la construcción de las variables usadas en los análisis. En el siguiente se expone y justifica la metodología econométrica utilizada. El cuarto epígrafe comenta los resultados de las estimaciones, tanto para la formación financiada como para la no financiada, y, finalmente, se termina con un resumen de las principales conclusiones. 


\section{DATOS Y VARIABLES}

Los datos utilizados en las estimaciones han sido extraídos de las olas segunda a séptima del PHOGUE (INE, 1995-2000). La razón que impide aprovechar la información contenida en el primer ciclo de la encuesta es que en ella no consta ni el tipo de contrato que une al trabajador con su empresa ni el tamaño del establecimiento, siendo ambas variables importantes determinantes en el modelo que se estima. Una vez eliminadas aquellas observaciones con valores perdidos en alguna de las variables, quedan 22.272 casos con información completa disponibles para estimar. Respecto a la variable dependiente, consideramos que existe formación cuando el individuo responde haber realizado algún tipo de curso de formación profesional u ocupacional durante el año de referencia ${ }^{2}$. Posteriormente, distinguimos entre los trabajadores cuyos cursos fueron pagados por la empresa (formación financiada) de aquéllos que no lo fueron (formación no financiada). La distribución según el sexo y el tipo de formación recibida, tanto de su incidencia como de su duración se muestra en la Tabla 1.

\section{TABLa 1.-Distribución de la incidencia a y duración del tipo de formación recibida} por sexo

\begin{tabular}{|l|c|c|c|}
\hline \multicolumn{1}{|c|}{ Tipo } & Hombres & Mujeres & Total \\
\hline Sin formación & $85,79 \%$ & $80,05 \%$ & $83,79 \%$ \\
\hline Con formación & $14,21 \%$ & $19,95 \%$ & $16,21 \%$ \\
\hline Financiada por el empleado & $5,42 \%$ & $9,98 \%$ & $7,01 \%$ \\
\hline $\begin{array}{l}\text { Duración media (días) } \\
\text { (Desviación típica) }\end{array}$ & 204,42 & 212,06 & 208,10 \\
\cline { 2 - 4 } & $(173,15)$ & $(173,77)$ & $(173,45)$ \\
\hline Financiada por el empleador & $8,79 \%$ & $9,97 \%$ & $9,20 \%$ \\
\hline $\begin{array}{l}\text { Duración media (días) } \\
\text { (Desviación típica) }\end{array}$ & 61,12 & 68,19 & 63,80 \\
\cline { 2 - 4 } & $(126,59)$ & $(118,81)$ & $(123,72)$ \\
\hline
\end{tabular}

${ }^{a}$ Para el cálculo de los porcentajes de asalariados con formación se tienen en cuenta aquéllos en los que la duración del curso es desconocida.

${ }^{2}$ Lamentablemente, el PHOGUE no proporciona información sobre la temática de los cursos y hasta qué punto se adaptan a los requerimientos del empleo. Sin embargo, la práctica totalidad de los trabajadores (un 98\%) responde que una de las razones que les llevó a realizarlos fue mejorar sus habilidades o expectativas laborales, lo que refleja su naturaleza de inversión profesional. 
En primer lugar, destaca el hecho de que en el período considerado sólo un 16,21\% de los asalariados había recibido formación en los últimos meses. En comparación con la evidencia de otros países el dato contrasta, por ejemplo, con el 34\% obtenido por Arulampalam y Booth (1998) a partir del Panel de Hogares Británico (BHPS). Adicionalmente, los datos disponibles de la OCDE (1999) muestran que los países del sur de Europa (España, Portugal, Italia...) tienen tasas de recepción de formación específica por parte de los asalariados relativamente bajas, comparados con los países del centro y el norte europeos (Reino Unido, Francia, Suecia...). En cuanto a la financiación de estos cursos en España, es más común que corresponda al empleador $(9,20 \%)$ que al empleado $(7,01 \%)$, aunque la diferencia no es notable. Ahora bien, la duración de los cursos realizados es muy superior en este último caso, triplicando el número de días de los cursos ofertados por las empresas, que quizás encuentran demasiado oneroso liberar a sus trabajadores durante mucho tiempo de sus funciones productivas. En cualquier caso, esta diferencia parece confirmar la pertinencia de analizar la duración por separado para estos dos tipos de formación.

Por otra parte, el examen por sexo permite constatar que existe variación tanto en la incidencia de la formación (casi duplicando la proporción femenina a la masculina en el caso de la formación autofinanciada), como en lo que se refiere a la duración de los cursos (con una duración media para las mujeres un $12 \%$ superior a la masculina en la formación financiada por la empresa).

En cuanto a las variables utilizadas en los análisis, se consideran como determinantes de la formación, además del sexo del trabajador, variables de capital humano y estado civil como características personales del mismo, junto a características del empleo y otras variables de control. Dentro de la primera categoría, se incluye el nivel de estudios del trabajador, la experiencia laboral medida en años ${ }^{3}$ y tres indicadores de desajuste educativo. Éstos se construyen a partir de dos preguntas de la encuesta sobre apreciaciones subjetivas del trabajador. En la primera, se le pregunta si sus conocimientos le permitirían realizar un trabajo más cualificado que el que desarrolla en la actualidad (lo que puede interpretarse como percepción de estar sobreeducado), mientras que la segunda es sobre si sus estudios le han proporcionado los conocimientos suficientes para realizar sus labores (lo que en caso de negativa puede interpretarse en principio como indicador de infraeducación). Como hay individuos que se declaran

3 Dado que el PHOGUE no permite obtener información completa sobre la vida laboral de los encuestados, ésta es una variable de experiencia potencial, medida como el tiempo desde que el individuo entró en el mercado laboral hasta el año corriente. 
simultáneamente sobreeducados e infraeducados, incluir en las estimaciones las dos variables tal y como aparecen en el PHOGUE puede llevar a confundir realidades distintas. Así, siguiendo la línea de Aguilar y García (2008), se cruzan ambas variables en cuatro categorías, siendo la referencia los trabajadores sin desajuste educativo. Dentro de los que podrían hacer un trabajo más cualificado (sobreeducados) se distingue entre aquellos que tienen formación suficiente para realizar el suyo actual (sobreeducados tipo 1) y los que no (sobreeducados tipo 2). Finalmente, aquellos con formación insuficiente para su trabajo o para otro más cualificado se incluyen en una tercera categoría (infraeducados).

Entre las características del empleo, se incluyen en primer lugar una variable ficticia para aquellos que no tienen un empleo indefinido ${ }^{4}$, otra para los trabajadores con menos de 3 años de antigüedad y la interacción entre ambas. En segundo lugar, se introducen indicadores para las distintas ocupaciones (10 categorías), sectores de actividad (11 categorías), tamaño del establecimiento (menos de 20 trabajadores, de 20 a 99 y de 100 o más) y empleo a tiempo parcial. Finalmente, se incluye un conjunto de ficticias temporales, que sirven para controlar la posible existencia de diferencias en los patrones de adquisición de formación a lo largo del tiempo, y una variable que recoge la tasa de paro regional ${ }^{5}$ para captar la influencia del estado del mercado de trabajo.

En la Tabla A1 del anexo se incluye información descriptiva sobre estas variables según las tres categorías de formación consideradas (sin formación, formación financiada y formación no financiada). Su observación muestra que la mayor parte de los individuos que reciben formación financiada son universitarios o tienen un diploma de segundo nivel de enseñanza secundaria, especialmente en el caso de las mujeres. Complementariamente, los trabajadores con formación inferior a la requerida para su empleo realizan menos cursos tanto financiados por ellos mismos como por su empresa, estando en el otro extremo los sobreeducados tipo 1. Por su parte, los individuos que financian su formación son los que presentan una menor experiencia en promedio. Dentro de las características del empleo, se advierte que la mayor parte de los trabajadores a los que su empresa les dedica formación tienen un nivel ocupacional alto o

${ }^{4}$ Este grupo está compuesto mayoritariamente por asalariados con contrato temporal, aunque también hay una pequeña proporción de trabajadores sin contrato.

5 El PHOGUE no proporciona información sobre la comunidad autónoma de residencia, sino que divide a España en 8 regiones más amplias. Así, la tasa de paro mencionada se calcula como la media del paro en las distintas comunidades de cada región, ponderando por el número de activos. 
medio-alto, se dedican a actividades bancarias, comerciales o de sector público y trabajan en establecimientos de tamaño grande. En cambio, los trabajadores temporales y a tiempo parcial tienden a autofinanciarse cursos de formación, probablemente con la intención de mejorar su situación laboral.

\section{METOdología ECONOMÉTRICA}

Como hemos visto anteriormente, apenas una pequeña proporción de la muestra de asalariados realiza actividades formativas, por lo que para una gran mayoría de los individuos de la base de datos la duración de las mismas se iguala a 0 días. Desde la perspectiva econométrica, esto plantea un problema de censura en los datos que sesgaría las estimaciones MCO realizadas en la muestra completa ${ }^{6}$. Para evitarlo, se propone como solución la estimación conjunta de la incidencia y la duración de la formación mediante un modelo tobit, en particular con efectos aleatorios ${ }^{7}$, cuya estimación por máxima verosimilitud produce estimadores con propiedades deseables. Además, estas estimaciones permiten conocer dos efectos complementarios. En primer lugar, ver cómo afectan las distintas variables explicativas a la probabilidad de recibir formación o invertir en ella y, en segundo lugar, para el caso en que exista formación, cuánto influyen esas variables en la propia duración de la misma. Para definir este modelo, denominamos a la variable de duración observada para el individuo i en el ciclo t del PHOGUE como $d_{i t}$, siendo ésta 0 si no existe formación, y partimos de una variable latente $d_{i t}^{*}$, que se observa si $d_{i t}^{*}>0$, y no si $d_{i t}^{*} \leq 0$. A partir de ella, podemos definir $d_{i t}$ como:

$$
d_{i t}=\left\{\begin{array}{ll}
d_{i t}^{*}=\beta^{\prime} x_{i t}+u_{i}+\epsilon_{i t} & \text { si } d_{i t}^{*}>0 \\
0 & \text { si } d_{i t}^{*} \leq 0
\end{array} \quad(i=1, \ldots N) ; \quad\left(t=1, \ldots T_{i}\right),\right.
$$

${ }^{6}$ Esto es así porque el término de error del modelo deja de tener esperanza nula y no sigue una distribución normal estándar, incumpliéndose por tanto las propiedades de los estimadores MCO. Tobin (1958) fue el primero en analizar este problema en el campo de la econometría, dando así nombre al modelo tobit.

7 Se propone que el modelo tobit sea con efectos aleatorios, dado que al disponer de una muestra de panel es posible tener en cuenta el efecto de la heterogeneidad inobservable entre los individuos analizados. Una metodología similar para estimar la duración de la formación puede encontrarse, entre otros trabajos, en Booth y otros (2003) y Malo y Sánchez (2005). 
lo que representa el modelo tobit a estimar. Como se constata en él, la variable latente $d_{i t}^{*}$ determina, en caso de ser positiva, que una observación pertenece al grupo de los trabajadores con formación, así como la duración de ésta (medida en días), y su valor coincide con el observado. De tomar $d_{i t}^{*}$ un valor nulo o negativo, la observación está censurada y $d_{i t}=0$. Puede verse a $d_{i t}^{*}$ como un indicador de la propensión a invertir en formación relacionado con los incentivos del trabajador y de la empresa, que determinan una inversión efectiva y la duración correspondiente si se supera cierto umbral; $x_{i t}$ y $\beta$ son, respectivamente, vectores de los valores de los distintos regresores y los coeficientes asociados, que determinan la esperanza condicional de $d_{i t}^{*} ; u_{i}$ y $\epsilon_{i t}$ son dos términos de error de distribución normal, independientes entre sí y de las variables incluidas en $x_{i t}$. La diferencia entre ambos es que mientras que $\epsilon_{i t}$ varía tanto de individuo a individuo como entre períodos temporales, $u_{i}$ presenta un valor constante en todas las observaciones de cada individuo. La inclusión de este término de error adicional, por tanto, permite contemplar la influencia de características no observadas de los individuos, constantes en el tiempo, en la determinación de la duración de los cursos de aprendizaje que realizan.

Por otra parte, la metodología ha de ser adaptada de algún modo para considerar por separado la formación dependiendo de quién la financie. En el caso de un modelo tobit, el procedimiento elegido consiste en estimar de forma separada por máxima verosimilitud dos modelos tobit con efectos aleatorios para cada sexo, uno para la duración de la formación financiada por el trabajador y otro para la financiada por la empresa. En cada uno de los casos, se eliminan las observaciones en las que existe formación del tipo contrario al considerado.

Como se ha indicado, tras estimar las distintas especificaciones, es posible calcular numéricamente dos conjuntos de efectos marginales complementarios, lo que facilita la interpretación de los resultados. El primero proporciona el efecto de cada variable en la incidencia de la formación (es decir, la probabilidad de que la empresa o el trabajador decidan invertir en este tipo de capital humano) y el segundo mide la influencia sobre la duración (es decir, la cantidad de tiempo que están dispuestos a dedicar a tal inversión), condicionada a que ésta sea positiva. Concretamente, para las dos únicas variables continuas en los modelos (experiencia laboral y tasa de paro regional), el efecto marginal se calcula como la derivada de la función correspondiente $\left(P\left(d_{i t}^{*}>0\right) \mathrm{o}\right.$ $\left.E\left(d_{i t}^{*} \mid d_{i t}^{*}>0\right)\right)$ respecto a cada variable, evaluada en la media muestral. Para el resto de variables, al tratarse de indicadores binarios, se utiliza el incremento en esas funciones al cambiar la variable de 0 a 1 , manteniendo el resto de determinantes de nuevo en sus valores medios. 


\section{Resultados}

Las Tablas 2 y 3 muestran, para la formación no financiada y la financiada, respectivamente ${ }^{8}$, los efectos marginales estimados de las principales variables utilizadas. Adicionalmente, también se incluye, al final de cada tabla, el valor medio de las estimaciones para la probabilidad de recibir formación y su duración, lo que permite conocer el valor relativo de los efectos marginales reseñados.

\subsection{Formación no financiada}

Los resultados de este tipo de formación para hombres y mujeres aparecen en la Tabla 2, donde se observan comportamientos bien diferenciados según el sexo, como muestran las predicciones medias obtenidas y las diferencias apreciables constatadas de los distintos determinantes para ambos colectivos. Respecto a las primeras, destaca el hecho de que el modelo asigna a las mujeres una probabilidad media en torno al $7 \%$ de adquirir formación por su cuenta, una cifra que se reduce a menos de la mitad en el caso de los hombres. Sin embargo, esta diferencia es de menor magnitud en cuanto a la duración de estos cursos, que es sólo 13 días superior en promedio para las mujeres (apenas un 10\% por encima del resultado para los hombres).

TABLA 2.-Modelo tobit de efectos aleatorios para la duración de la formación no financiada. Efectos marginales

\begin{tabular}{|l|cc|cc|cc|cc|}
\hline & \multicolumn{3}{|c|}{$\begin{array}{c}\text { Efecto sobre probabilidad } \\
\text { de duración }>0\end{array}$} & \multicolumn{3}{c|}{$\begin{array}{c}\text { Efecto sobre duración } \\
\text { si duración }>0\end{array}$} \\
\hline Variable & \multicolumn{2}{|c|}{ Hombres } & \multicolumn{2}{|c|}{ Mujeres } & \multicolumn{2}{c|}{ Hombres } & \multicolumn{2}{c|}{ Mujeres } \\
\hline Nivel educativo & & & & & & & & \\
\hline $1^{\mathrm{er}}$ nivel secundaria & 0,0136 & $* *$ & 0,0738 & $* * *$ & 8,8996 & $* *$ & 27,6963 & $* * *$ \\
\hline FP1 & 0,0770 & $* * *$ & 0,1658 & $* * *$ & 37,3408 & $* * *$ & 54,4457 & $* * *$ \\
\hline $2^{\circ}$ nivel secundaria & 0,0368 & $* * *$ & 0,1305 & $* * *$ & 20,6655 & $* * *$ & 44,5514 & $* * *$ \\
\hline FP2 & 0,1027 & $* * *$ & 0,2094 & $* * *$ & 46,8876 & $* * *$ & 66,8753 & $* * *$ \\
\hline
\end{tabular}

${ }^{8}$ En la Tablas A2 y A3 del Anexo se muestra el valor de los coeficientes estimados junto a los correspondientes errores estándar en los modelos para la formación no financiada y financiada, respectivamente. 
TABLA 2 (cont.).-Modelo tobit de efectos aleatorios para la duración de la formación no financiada. Efectos marginales

\begin{tabular}{|c|c|c|c|c|c|c|c|c|}
\hline \multirow{3}{*}{$\begin{array}{l}\text { Variable } \\
\text { Diplomado }\end{array}$} & \multicolumn{4}{|c|}{$\begin{array}{c}\text { Efecto sobre probabilidad } \\
\text { de duración }>0\end{array}$} & \multicolumn{4}{|c|}{$\begin{array}{l}\text { Efecto sobre duración } \\
\text { si duración }>0\end{array}$} \\
\hline & \multicolumn{2}{|c|}{ Hombres } & \multicolumn{2}{|c|}{ Mujeres } & \multicolumn{2}{|c|}{ Hombres } & \multicolumn{2}{|c|}{ Mujeres } \\
\hline & 0,0489 & $* * *$ & 0,1053 & $* * *$ & 25,6451 & $* * *$ & 36,7777 & $* * *$ \\
\hline Licenciado & 0,0321 & $* *$ & 0,1173 & $* * *$ & 18,2301 & $* * *$ & 40,4568 & $* * *$ \\
\hline Vive en pareja & $-0,0078$ & $* *$ & $-0,0330$ & $* * *$ & $-5,2701$ & ** & $-13,5284$ & *** \\
\hline Experiencia & $-0,0014$ & $* * *$ & $-0,0025$ & $* * *$ & $-0,9636$ & $* * *$ & $-1,0425$ & $* * *$ \\
\hline Temporal & $-0,0122$ & * & 0,0042 & & $-8,6415$ & $*$ & 1,7367 & \\
\hline \multicolumn{9}{|l|}{ Antigüedad } \\
\hline Menos de 3 años & 0,0128 & $* * *$ & 0,0433 & $* * *$ & 8,7082 & $* * *$ & 17,8858 & $* * *$ \\
\hline Temporal y menos de tres años & 0,0353 & $* * *$ & 0,0217 & & 21,6293 & $* * *$ & 8,8525 & \\
\hline \multicolumn{9}{|l|}{ Ocupación } \\
\hline Directivo & $-0,0011$ & & 0,0723 & & $-0,7711$ & & 25,5724 & \\
\hline Profesional & 0,0083 & & 0,0354 & $*$ & 5,3732 & & 13,6941 & * \\
\hline Técnico & 0,0076 & & 0,0427 & $* *$ & 4,9557 & & 16,1594 & $* *$ \\
\hline Oficinistas & 0,0111 & & 0,0382 & ** & 7,0875 & & 14,7875 & $* * *$ \\
\hline Dependientes, seguridad & $-0,0096$ & $* *$ & 0,0381 & $* * *$ & $-7,1825$ & $*$ & 14,8998 & $* * *$ \\
\hline Cualificado agricultura & $-0,0078$ & & 0,2236 & * & $-5,8290$ & & 69,3566 & ** \\
\hline Cualificado industria & $-0,0002$ & & 0,0379 & & $-0,1368$ & & 14,3745 & * \\
\hline Operadores & 0,0029 & & 0,0218 & & 1,9664 & & 8,5354 & \\
\hline Fuerzas armadas & $-0,0091$ & & 0,1596 & & $-6,9743$ & & 51,2786 & \\
\hline Tiempo parcial & 0,0462 & $* * *$ & 0,0094 & & 24,2247 & $* * *$ & 3,8164 & \\
\hline \multicolumn{9}{|l|}{ Tamaño del establecimiento } \\
\hline Menos de 20 trabajadores & $-0,0034$ & & 0,0172 & ** & $-2,3511$ & & 7,1387 & $* *$ \\
\hline 20 a 99 trabajadores & 0,0053 & & 0,0186 & ** & 3,5928 & & 7,5273 & $* *$ \\
\hline \multicolumn{9}{|l|}{ Desajuste educativo } \\
\hline Sobreeducado 1 & 0,0080 & $* *$ & 0,0052 & & 5,3714 & $* *$ & 2,1628 & \\
\hline Sobreeducado 2 & $-0,0032$ & & 0,0016 & & $-2,2766$ & & 0,6771 & \\
\hline Infraeducado & $-0,0235$ & $* * *$ & $-0,0323$ & $* * *$ & $-18,3230$ & $* * *$ & $-14,5058$ & $* * *$ \\
\hline Predicción media & 0,0305 & & 0,0711 & & 151,5139 & & 164,3760 & \\
\hline
\end{tabular}

Notas: El individuo de referencia es un trabajador con estudios primarios o sin estudios, con contrato indefinido y 3 o más años de antigüedad en la empresa, que realiza un empleo no cualificado en el sector industrial a tiempo completo en un establecimiento de 100 o más empleados, sin desajuste educativo y es entrevistado en 1995.

Se incluyen controles adicionales de sector de actividad, año y tasa de paro regional.

$* * *$ Efecto marginal significativo al 1\%, ** al 5\%, * al $10 \%$. 
El detalle de los determinantes señala, en primer lugar, que el nivel educativo tiene una relación positiva con la variable dependiente. Así, la probabilidad de que un trabajador (sea hombre o mujer) adquiera formación laboral y que ésta sea de mayor duración, aumenta con el nivel educativo, tanto de tipo general ${ }^{9}$ como aún más de tipo profesional, alcanzando el máximo entre los titulados de FP2. Este resultado es común en la literatura (Greenhalg y Stewart, 1987; Mincer, 1988; Altonji y Spletzer, 1991; para el caso español, Caparrós y otros, 2004) y puede explicarse porque las mismas características que permiten adquirir educación formal reducen el coste de la inversión en este otro tipo de formación, al incrementar la capacidad del individuo para aprovechar estudios posteriores. El hecho de que tanto la probabilidad como la duración sean más elevadas para los titulados de formación profesional probablemente se deba a la predominancia de contenidos prácticos en estos estudios, sujetos a niveles relativamente altos de obsolescencia que han de paliarse a través de cursos específicos (ver Booth y otros, 2003, y Almeida Santos y Mumford, 2005, para una evidencia similar relativa al mercado británico y utilizando métodos alternativos). Además, los efectos marginales en todas las categorías son superiores para las mujeres que para los hombres, en una magnitud que en general ronda los 10 puntos porcentuales para la probabilidad, o los 20 días para la duración.

Complementariamente, el efecto del desajuste educativo indica que son los trabajadores, de ambos sexos, con formación inferior a la requerida para su empleo los que tienen menor probabilidad de realizar cursos por su cuenta (aproximadamente un 3\% menos), siendo éstos además de inferior duración (unos 15-18 días menos), mientras que para el grupo de los que se consideran capaces de realizar tareas de cualificación superior (sobreeducados 1) son los hombres los que hacen más cursos y de duración 5 días mayor. Al parecer, los sobreeducados pueden pensar que invertir en formación práctica para la empresa les ayudará a alcanzar un puesto acorde con sus cualificaciones, compensándoles así el sacrificio de adquirir formación.

En lo que se refiere a la experiencia laboral acumulada, se constata una significativa asociación negativa con la cantidad de formación que los trabajadores de ambos sexos financian. Este resultado concuerda con las predicciones teóricas, ya que al aumentar la edad del trabajador se reduce el lapso de tiempo en el que recuperar cualquier inversión en capital humano y, por tanto, el incentivo a realizarla (Jonker y de Grip, 1999, con datos holandeses, además de los estudios ya citados, encuentran resultados similares).

9 A excepción de los estudios de licenciatura. 
Una variable cuyo efecto difiere de forma importante entre ambos sexos es la que capta la importancia de las responsabilidades familiares, medida por el estado civil. Así, para los dos colectivos, se comprueba que el vivir en pareja reduce de forma significativa tanto la probabilidad como el tiempo dedicado a realizar formación por cuenta propia. Sin embargo, la magnitud de este efecto es más del doble para las mujeres que para los hombres. Esto sugiere que el hecho de estar casado conlleva unas responsabilidades que limitan el tiempo susceptible de dedicarse a inversiones en capital humano y que esa reducción es más acusada entre las mujeres, pues son ellas las que cargan con una parte mayor de las tareas del hogar.

También en el tipo de contrato y la antigüedad laboral se producen divergencias claras entre hombres y mujeres. En ambos casos, la probabilidad de adquirir formación es mayor entre los trabajadores con menos de tres años de antigüedad; sin embargo, entre los hombres este efecto se ve complementado si el individuo está vinculado a la vez a la empresa con un contrato temporal. Esto sugiere que los trabajadores con menor antigüedad necesitan posicionarse como productivos ante sus empleadores para afianzar su posición. Este motivo es aún más acuciante para los que tienen un contrato temporal, ya que entonces el riesgo de despido es todavía mayor y, además, el tránsito hacia el empleo permanente en el mercado español suele realizarse en los primeros tres años de relación con la empresa (Amuedo, 2000; Güell y Petrongolo, 2000).

Otra característica importante del empleo, el tipo de jornada, tiene un efecto diferenciado por género en la formación adquirida, ya que si los varones efectúan la jornada a tiempo parcial aumenta la probabilidad de adquirir formación en casi 5 puntos porcentuales, cuando la misma característica no ejerce influencia en la formación de las asalariadas. Lo esperable sería que a mayor tiempo disponible, liberado por una menor cantidad de tareas laborales, aumentaran las facilidades para realizar formación y el tiempo dedicado a ésta. El que no se cumpla esta relación para las mujeres parece sugerir que el tiempo que las asalariadas con este tipo de jornada no emplean en el mercado de trabajo lo dedican a tareas de otra naturaleza, probablemente relacionadas con sus responsabilidades familiares. Lo contrario ocurre en el caso de los hombres, que entienden el empleo a tiempo parcial como una etapa a aprovechar para adquirir formación con vistas a pasar a jornada completa. Para las mujeres, la elección de este tipo de empleos parece responder más a una limitación voluntaria en su oferta laboral.

Finalmente, el tamaño del centro de trabajo no ejerce efecto significativo alguno entre los hombres, y sí tiene una influencia positiva no desdeñable para las mujeres que trabajan en empresas pequeñas o medianas (hasta 100 trabajadores), para quienes es más probable que adquieran formación por su cuenta y lo hacen durante unos 7 días más. 


\subsection{Formación financiada}

Un primer interrogante en el caso de los cursos ofrecidos por las empresas es saber si difieren entre hombres y mujeres. En general, la literatura empírica suele encontrar evidencia de que las mujeres reciben menos formación financiada que los hombres. Así, por ejemplo, Veum (1993), Jonker y de Grip (1999) y Pischke (2001) para Estados Unidos, Holanda y Alemania, respectivamente, descubren una menor duración de los cursos proporcionados a las mujeres, independientemente de la probabilidad que tengan para ser seleccionadas. Sin embargo, nuestros datos (Tabla 3) indican que, para una mujer, la probabilidad media de recibir formación financiada por su empresa es de alrededor del 6\%, algo mayor que el $5 \%$ estimado para los hombres, aunque la duración de los cursos es prácticamente igual para ambos. Aún así, los resultados muestran diferencias importantes en el signo y magnitud de varios regresores, justificando la estimación por separado para hombres y mujeres.

El análisis detallado de estos determinantes refleja de nuevo una influencia positiva del nivel de educación formal en la probabilidad de recibir formación financiada y en la duración de los cursos. Sin embargo, ahora el efecto es mayor en las enseñanzas de tipo general (observándose el máximo para los licenciados y diplomados, seguidos del segundo nivel de secundaria) que en los estudios profesionales, y esto para ambos sexos. Este efecto complementario entre ambos tipos de formación laboral según el nivel de educación inicial (general o vocacional) aparece también en los estudios empíricos ya citados. Esta relación creciente entre nivel educativo y formación financiada sugiere que el mercado laboral amplifica las divergencias de partida entre los trabajadores, proporcionando mejores oportunidades a aquellos con mayor nivel educativo. Por otra parte, los resultados muestran que el efecto de la educación es mucho más acusado para las mujeres, sobre todo en los estudios de tipo general, puesto que reciben el doble de días de formación que los hombres y tienen más del doble de probabilidad de recibirlos. A título de ilustración, las licenciadas tienen una probabilidad 14 puntos porcentuales superior de recibir formación y lo hacen durante 23 días más que las de estudios primarios o inferiores, cuando estas cifras son para los licenciados de 5 puntos y 9 días, respectivamente. Al parecer, un título educativo superior transmite al empresario una señal positiva sobre la vinculación de la trabajadora con el empleo, ya que las tasas de actividad de las universitarias son equiparables a las masculinas (ver, por ejemplo, De la Rica y otros, 2005). 
TABla 3.-Modelo Tobit de Efectos Aleatorios para la duración de la Formación Financiada. Efectos Marginales

\begin{tabular}{|c|c|c|c|c|c|c|c|c|}
\hline \multirow[b]{2}{*}{ Variable } & \multicolumn{4}{|c|}{$\begin{array}{c}\text { Efecto sobre probabilidad } \\
\text { de duración }>0\end{array}$} & \multicolumn{4}{|c|}{$\begin{array}{l}\text { Efecto sobre duración } \\
\text { si duración }>0\end{array}$} \\
\hline & \multicolumn{2}{|c|}{ Hombres } & \multicolumn{2}{|c|}{ Mujeres } & \multicolumn{2}{|c|}{ Hombres } & \multicolumn{2}{|c|}{ Mujeres } \\
\hline \multicolumn{9}{|l|}{ Nivel educativo } \\
\hline $1^{\mathrm{er}}$ nivel secundaria & 0,0234 & $* * *$ & 0,0613 & $* * *$ & 5,1763 & $* * *$ & 11,3835 & *** \\
\hline FP1 & 0,0525 & $* * *$ & 0,0717 & $* * *$ & 10,3496 & $* * *$ & 12,5086 & *** \\
\hline $2^{\circ}$ nivel secundaria & 0,0530 & $* * *$ & 0,1386 & $* * *$ & 10,5510 & $* * *$ & 22,5314 & *** \\
\hline FP2 & 0,0846 & $* * *$ & 0,1282 & $* * *$ & 15,6713 & $* * *$ & 20,6482 & *** \\
\hline Diplomado & 0,0875 & $* * *$ & 0,1344 & $* * *$ & 15,9948 & *** & 21,9708 & *** \\
\hline Licenciado & 0,0458 & $* * *$ & 0,1408 & $* * *$ & 9,2047 & $* * *$ & 22,8122 & *** \\
\hline Vive en pareja & 0,0173 & $* * *$ & 0,0140 & $* * *$ & 4,1731 & $* * *$ & 2,9381 & $* *$ \\
\hline Experiencia & $-0,0009$ & $* * *$ & 0,0001 & & $-0,2077$ & **** & 0,0222 & \\
\hline Temporal & $-0,0333$ & $* * *$ & 0,0009 & & $-8,1668$ & $* * *$ & 0,1843 & \\
\hline \multicolumn{9}{|l|}{ Antigüedad } \\
\hline Menos de 3 años & $-0,0232$ & $* * *$ & $-0,0249$ & $* * *$ & $-5,5347$ & $* * *$ & $-5,2229$ & *** \\
\hline Temporal y menos de tres años & 0,0283 & $* *$ & 0,0017 & & 6,1999 & $* *$ & 0,3598 & \\
\hline \multicolumn{9}{|l|}{ Ocupación } \\
\hline Directivo & 0,0830 & $* * *$ & 0,1666 & $* * *$ & 15,1700 & $* * *$ & 25,3384 & *** \\
\hline Profesional & 0,0424 & $* * *$ & 0,0773 & $* * *$ & 8,5815 & $* * *$ & 13,7278 & *** \\
\hline Técnico & 0,0453 & $* * *$ & 0,0795 & $* * *$ & 9,1368 & $* * *$ & 13,7761 & *** \\
\hline Oficinistas & 0,0552 & $* * *$ & 0,0544 & $* * *$ & 10,8090 & **** & 10,0399 & *** \\
\hline Dependientes, seguridad & 0,0334 & $* *$ & 0,0637 & $* * *$ & 6,9796 & $* * *$ & 11,6998 & *** \\
\hline Cualificado agricultura & $-0,0038$ & & 0,1461 & & $-0,8914$ & & 22,5781 & \\
\hline Cualificado industria & 0,0141 & & $-0,0201$ & & 3,1893 & & $-4,5388$ & \\
\hline Operadores & 0,0050 & & 0,0236 & & 1,1486 & & 4,5213 & \\
\hline Fuerzas armadas & 0,0386 & & 0,0874 & & 7,7623 & & 14,5214 & \\
\hline Tiempo parcial & 0,0088 & & $-0,0207$ & $* * *$ & 1,9648 & & $-4,5976$ & $* *$ \\
\hline \multicolumn{9}{|l|}{ Tamaño del establecimiento } \\
\hline Menos de 20 trabajadores & $-0,0293$ & $* * *$ & $-0,0421$ & $* * *$ & $-6,8999$ & $* * *$ & $-8,8161$ & *** \\
\hline 20 a 99 trabajadores & $-0,0158$ & $* * *$ & $-0,0148$ & $* *$ & $-3,8190$ & $* * *$ & $-3,1471$ & $* *$ \\
\hline \multicolumn{9}{|l|}{ Desajuste educativo } \\
\hline Sobreeducado 1 & 0,0066 & & 0,0084 & & 1,5175 & & 1,7235 & \\
\hline Sobreeducado 2 & $-0,0102$ & $* *$ & 0,0020 & & $-2,4307$ & $*$ & 0,4197 & \\
\hline
\end{tabular}


Tabla 3 (cont.).- Modelo Tobit de Efectos Aleatorios para la duración de la Formación Financiada. Efectos Marginales

\begin{tabular}{|c|c|c|c|c|c|c|c|c|}
\hline \multirow{3}{*}{$\begin{array}{l}\text { Variable } \\
\text { Infraeducado }\end{array}$} & \multicolumn{4}{|c|}{$\begin{array}{c}\text { Efecto sobre probabilidad } \\
\text { de duración }>0\end{array}$} & \multicolumn{4}{|c|}{$\begin{array}{l}\text { Efecto sobre duración } \\
\text { si duración }>0\end{array}$} \\
\hline & \multicolumn{2}{|c|}{ Hombres } & \multicolumn{2}{|c|}{ Mujeres } & \multicolumn{2}{|c|}{ Hombres } & \multicolumn{2}{|c|}{ Mujeres } \\
\hline & $-0,0195$ & $* * *$ & $-0,0357$ & $* * *$ & $-4,7859$ & $* * *$ & $-8,2835$ & $* * *$ \\
\hline Predicción media & 0,0515 & & 0,0605 & & 74,2316 & & 73,6081 & \\
\hline
\end{tabular}

Notas: El individuo de referencia es un trabajador con estudios primarios o sin estudios, con contrato indefinido y 3 o más años de antigüiedad en la empresa, que realiza un empleo no cualificado en el sector industrial a tiempo completo en un establecimiento de 100 o más empleados, sin desajuste educativo y es entrevistado en 1995.

Se incluyen controles adicionales de sector de actividad, año y tasa de paro regional.

$* * *$ Efecto marginal significativo al 1\%, ** al 5\%, * al 10\%.

Como complemento a las variables de educación, el desajuste educativo tiene un considerable efecto negativo en el caso de los infraeducados. Así, en comparación con los que han conseguido equiparar su formación con los requerimientos de su empleo, aquellos que no tienen un nivel educativo suficiente para ejercer el puesto que ocupan tienen una probabilidad entre 2 y 3,6 puntos inferior de recibir formación por parte de sus empresas, y de una duración de 5 y 8 días menor, según se trate, respectivamente, de hombres o mujeres. Este resultado parece indicar que aquellos que han alcanzado una posición superior a la que en principio les permitiría su educación encontrarían demasiadas dificultades para asimilar los conocimientos y seguir ascendiendo, por lo que a las empresas no les resultaría rentable proporcionarles formación, dado el elevado coste que ello les supondría. En el caso de los hombres, los denominados «sobreeducados $2 \gg^{10}$ también reciben una ligera penalización tanto en términos de probabilidad de ser elegidos por sus empresas como, en caso afirmativo, en la duración de los cursos. Esto puede parecer contradictorio, en la medida en que los trabajadores sin formación inicial adecuada serían los más necesitados de este tipo de inversión, aunque dada la naturaleza de los datos es posible que este desajuste inicial esté ya corregido. Lo que probablemente sucede es que las empresas consideran la falta de un título adecuado para el puesto como un indicador de que inversiones ulteriores en formación no resultarían especialmente rentables, sobre todo si se hacen con vistas a un posible ascenso. Por su parte, a los sobreeducados de ambos sexos que se consideran con formación

10 Es decir, aquellos con titulación suficiente para hacer trabajos más cualificados pero que sin embargo no fueron preparados para la ocupación que desempeñan. 
suficiente para realizar su trabajo (sobreeducados 1), las empresas no les dedican recursos suplementarios a su formación, pues no parece necesario hacerlo ni tampoco les reportaría beneficios.

En cuanto a la experiencia laboral, ésta tiene una influencia negativa para los hombres, como sucedía en el caso de la formación autofinanciada, lo que se explica a partir del menor tiempo restante que tiene la empresa para recuperar las inversiones realizadas. Resultados similares se presentan tanto en Jonker y de Grip (1999) como en Booth y otros (2003). Sin embargo, en el caso de las mujeres una mayor experiencia laboral no reduce la formación (cuando sí lo hacía para la formación autofinanciada) y contradice las predicciones teóricas. Es posible que las mujeres con mayor experiencia accedieran al mercado de trabajo cuando éste era eminentemente masculino, de modo que quizás se trate de un grupo especialmente autoseleccionado en el que predominen trabajadoras de alta calidad, lo que podría compensar el efecto negativo esperado de la experiencia en la rentabilidad de la formación.

El estado civil tiene un efecto contrario al observado para la formación no financiada, dedicando las empresas mayor cantidad de formación a los que viven en pareja, tanto si se trata de mujeres como de hombres, aunque el efecto es ligeramente superior para estos últimos. Esto sugiere cierta sustituibilidad entre los dos tipos de formación, con los trabajadores solteros financiándose unos cursos que sus empleadores no les proporcionan. Por otra parte, el tener una pareja estable quizás señale a la empresa un mayor grado de compromiso del trabajador con el empleo, indicándole que éste va a aprovechar en el futuro la formación que le otorga la empresa.

También se observa cierto grado de compensación entre inversiones en capital humano efectuadas por empresa y trabajador en los resultados de las variables de antigüedad y tipo de contrato. Así, mientras que antes eran los trabajadores temporales y con menos antigüedad los que en mayor medida adquirían formación por su cuenta, ahora el efecto es el contrario. Para los hombres, el grupo con menor probabilidad de recibir formación y de duración más corta es el de los temporales con más de tres años de antigüedad (3 puntos y 6 días menos, respectivamente, que los permanentes con similar antigüedad). Este resultado es consistente con la evidencia previa tanto para España (Caparrós y otros, 2004) como para el Reino Unido (Arulampalam y Booth, 1998), y se explica porque los trabajadores temporales pueden ser considerados por la empresa como un grupo poco estable, en la medida en que es más fácil y por lo tanto más probable que sean despedidos, lo que convierte a la formación en una inversión más arriesgada. Sin embargo, este efecto se ve corregido en cierta medida entre los trabajadores con menos antigüedad, ya que en ese caso la diferencia con los asalariados permanentes se reduce a 1 punto porcentual en la probabilidad y 2 días en la duración, 
debido al efecto positivo de la variable de interacción para los temporales con menos de 3 años de antigüedad. Al parecer, las empresas concentran la inversión formativa en los trabajadores con contrato permanente y más antigüedad, para evitar las pérdidas que supondría un abandono antes de recuperar el coste desembolsado y, en el caso de los trabajadores que llevan menos tiempo en la empresa, la formación se otorga como mecanismo para seleccionar a los más aptos para permanecer en ella, y así poder cambiarlos a permanentes si son eventuales. En cuanto a las mujeres los resultados son similares, salvo que el tipo de contrato no juega ningún papel significativo. Esta diferencia sugiere que las empresas no tienden a utilizar el contrato eventual para las recién llegadas como un instrumento en su tránsito a la permanencia.

Siguiendo con las características del empleo, la ocupación tiene, a grandes rasgos, una influencia similar en la probabilidad y duración de los cursos tanto para hombres como para mujeres. Esta relación, además, es parecida a la hallada anteriormente entre educación y formación, encontrándose los mayores efectos marginales entre los directivos, seguidos por profesionales, técnicos y trabajadores administrativos, que son las ocupaciones con mayor cualificación. Esto lleva a pensar que la actualización de conocimientos en esas tareas necesita de más dedicación por parte de las empresas, en la medida que son las que exigen mayor responsabilidad. En cuanto al tipo de jornada, ya se ha visto anteriormente que ésta es una característica especialmente vinculada a las diferencias entre hombres y mujeres, y su influencia sobre la formación financiada no es una excepción, aunque actúe de manera inversa a como lo hacía en la autofinanciada. Así, mientras que las empresas no varían la cantidad de formación en función del tipo de jornada que realicen sus asalariados, sí lo hacen con las mujeres, para quienes la probabilidad de recibir cursos se reduce en 2 puntos porcentuales y su duración en unos 5 días si la jornada es a tiempo parcial. En este caso, la explicación más plausible es que este tipo de asalariadas no tengan planes de pasar a jornada completa, de manera que inversiones en su capacitación por parte de sus empleadores no podrían ser rentabilizadas durante una jornada estándar.

Finalizando con otras características del empleo, el tamaño de la empresa tiene un efecto claro sobre la formación financiada y es similar para hombres y mujeres. Así, siendo la referencia las empresas con 100 trabajadores o más, a medida que disminuye su tamaño también lo hace la formación otorgada a los empleados y el tiempo que se le dedica a la misma, un resultado similar al encontrado, por ejemplo, por Booth y otros (2003). Hay varias explicaciones posibles para este hecho. Puede ser que sólo las grandes empresas dispongan de suficiente infraestructura y recursos como para proporcionar cursos de manera regular y duración considerable, ya sea en sus propias instalaciones o a través de su adquisición en una empresa especializada. Por otra parte, 
al aumentar el tamaño de una organización lo hace asimismo la cantidad de puestos de mayor responsabilidad a cubrir, lo que hace necesario el uso de algún procedimiento de formación y selección, para lo que ese tipo de cursos puede resultar conveniente.

\section{Conclusiones}

El objetivo de este trabajo ha sido presentar nueva evidencia empírica sobre los determinantes de la formación a lo largo de la vida laboral de los españoles, que es de especial importancia en un mercado que prima crecientemente el trabajo cualificado. Para ello, se han utilizado los datos individuales del PHOGUE (1995-2000), a partir de los cuales se han estimado diversos modelos tobit de efectos aleatorios para datos de panel. Esto permite tener en cuenta el efecto de los regresores sobre la probabilidad de realizar cursos de formación y también sobre la duración de tales cursos, mejorando la precisión con la que se investiga el fenómeno. Además, los modelos han sido estimados por separado para la formación financiada por las empresas, de una parte, y la financiada por los trabajadores, de otra.

Es destacable que los determinantes sean distintos dependiendo de quién financie la formación (empresa o asalariado). Así, por ejemplo, mientras que trabajar con un contrato precario reduce el incentivo empresarial para invertir en capital humano específico, entre los trabajadores temporales con menos antigüedad es más frecuente encontrar aquellos que, probablemente intentando estabilizarse, se financian en mayor medida este tipo de inversiones. Por el contrario, sea quién sea el que financie las actividades formativas, algunas de las variables con mayor influencia positiva, tanto en la incidencia como en la duración, son el nivel educativo, la categoría profesional y el tamaño de la empresa. Esto parece sugerir que la formación complementa a inversiones previas en capital humano y se concentra en los trabajadores con un mejor estatus laboral. Así, en la medida en que la formación repercuta en incrementos salariales, la formación tendería a amplificar las desigualdades.

En cuanto a las diferencias por género, cabe señalar que para los dos tipos de formación, el efecto positivo del nivel de educación, tanto sobre la probabilidad de recibir formación como sobre la duración de la misma si efectivamente se realiza es más acusado para las mujeres. Esto puede deberse a un intento por evitar la discriminación, proporcionando al empresario con esta mayor inversión en capital humano información positiva sobre la propia productividad, o a que las empresas perciban que el incremento en la productividad correspondiente al pasar de un título educativo inferior a otro superior sea mayor entre las mujeres que entre los hombres, debido a que niveles altos 
de educación suelen asociarse en las mujeres a un compromiso a largo plazo con el mercado de trabajo. Por otra parte, algunas diferencias por sexo son de especial interés. Así, mientras que para los hombres el trabajar a tiempo parcial aumenta la cantidad de formación financiada por cuenta propia, probablemente con vistas a pasar a jornada completa, esta característica no tiene efecto en el caso de las mujeres, sugiriendo que la elección de trabajar a tiempo parcial ha sido voluntaria, para tener más tiempo que dedicar a tareas familiares. Lo contrario ocurre, en cambio, para la formación financiada por la empresa. Los hombres con jornada reducida no se ven afectados por ella, mientras que las mujeres en tal situación reciben menos cursos por cuenta de sus empleadores y además son de menor duración, pues quizás éstos dudan de poder rentabilizar sus inversiones en estas jornadas reducidas.

\section{REFERENCIAS}

Acemoglu, D. y PischKe, Jörn S. (1999), «The Structure of Wages and Investment in Imperfect Labor Markets», Journal of Political Economy, 107(3), págs. 539-572.

Aguilar, Mª I. y García, D. (2008), «Desajuste Educativo y Salarios en España: Nueva Evidencia con Datos de Panel», Estadística Española, 50(168), págs. 393-426.

Albert, C.; García Serrano, C. y Hernanz, V. (2005a), «Firm-Provided Training and Temporary Contracts», Spanish Economic Review 7(1), págs. 67-88.

- (2005b), «Los Determinantes de la Formación en la Empresa y sus Rendimientos», Comunicación presentada en las VI Jornadas de Economía Laboral, Alicante, julio 2005.

Almeida, S. F. y Mumford, K. (2005), «Employee Training and Wage Compression in Britain», The Manchester School 73(3), págs. 321-342.

Altonji, J. y Spletzer, J. (1991), «Worker Characteristics, Job Characteristics, and the Receipt of On-the-Job Training», Industrial and Labor Relations Review, 45(1), págs. 58-79.

Amuedo, C. (2000), «Work Transitions into and out of Involuntary Temporary Employment in a Segmented Market: evidence from Spain», Industrial and Labour Relations Review, 53(2), págs. 309-325.

Arulampalam, W. y Bоoth, A. L. (1998), «Training and Labour Market Flexibility: Is there a Trade-Off?», British Journal of Industrial Relations, 36(4), págs. 521-536.

Becker, G. S. (1964), Human Capital: A Theoretical and Empirical Analysis, with Special Reference to Education, The University of Chicago Press, Chicago y Londres, 3. ${ }^{\text {a }}$ ed., 1993.

Blau, F. D. y Khan, L. M. (1996), «International Differences in Male Wage Inequality: Institutions versus Market Forces», Journal of Political Economy, 104(4), págs. 791-837. 
Booth, A. L.; Francesconi, M. y Zoega, G. (2003), «Unions, Work-Related Training, and Wages: Evidence for British Men», Industrial and Labor Relations Review, 57 (1), págs. 68-91. Caparrós, A.; Navarro, M. ${ }^{a}$ L. y Rueda, M. F. (2004), «Efectos de la temporalidad sobre la formación recibida durante el empleo», Cuadernos de economía, 27(74) págs. 51-73

Chang, Ch. y Wang, Y. (1995), «A Framework for Understanding Differences in Labor Turnover and Human Capital Investment», Journal of Economic Behavior and Organization, 28(1) págs. 91-105.

De la Rica, S.; Dolado, J. J. y Llorens, V. (2005), «Ceiling and Floors: Gender Wage Gaps by Education in Spain», IZA Discussion Paper núm. 1483.

García-Espejo, I. (1999), «Formación en el Trabajo y Movilidad Laboral», Papers 59, págs. 195-219.

Greenhalg, Ch. y Stewart, M. (1987), «The effects and Determinants of Training», Oxford Bulletin of Economics \& Statistics, 49(2), págs. 171-190.

Güell, M. y Petrolongo, B. (2000), «Workers' Transitions from Temporary to Permanent Employment: the Spanish Case», Centre for Economic Performance DP n 438.

Hashimoto, M. (1981), «Firm-Specific Human Capital as a Shared Investment», American Economic Review, 71(3), págs. 476-482.

Jonker, N. y Grip, A. de (1999), «Do Employees with Flexible Contracts Receive less Training?», Documento de trabajo, Universidad de Maastricht.

Katz, E. y Ziderman, A. (1990), «Investment in general training: The role of information and labour mobility», Economic Journal 100 (dic.), págs. 1147-1158.

Leuven, E. (2005), «The Economics of Private Sector Training: A Survey of the Literature», Journal of Economic Surveys, 19(1), págs. 91-111.

LYNCH, L. M. (1989), «Private sector training and its impact on the earnings of young workers», NBER WP n 2872.

Malo, M. A. y SÁnchez, N. (2005), «Training in Imperfectly Competitive Labour Markets: The Importance of the Scope of Collective Bargaining», Comunicación presentada en las VI Jornadas de Economía Laboral, Alicante, julio 2005.

Mincer, J. (1988), «Job training, Wage Growth, and Labor Turnover», NBER WP nº 2690.

OCDE (1999), «Training of Adult Workers in OECD Countries: Measurement and Analysis», Employment Outlook, junio, págs. 133-155. París.

Peraita, C. (2000), «Características de la Formación en la Empresa Española», Papeles de Economía Española, núm. 86, págs. 295-307.

Pischke, J. S. (2001), «Continuous Training in Germany», Journal of Population Economics, 14(3), págs. 523-548.

Rueda, M. F. (2006), Discriminación Salarial por Razón de Género y Capital Humano: Un Análisis con Datos de Panel, Tesis Doctoral. Universidad de Málaga. 
Salas-Velasco, M. (2007): «Graduates on the labor market: Formal and informal post-school training investments». Higher Education, núm. 54, págs. 227-245

Tobin, J. (1958), «Estimation of Relationships for Limited Dependent Variables», Econometrica 26, págs. 24-36.

Veum, J. R. (1995), «Sources of Training and their Impact on Wages», Industrial and Labor Relations Review, 48(4), págs. 812-826. 


\section{ANEXO}

TABLA A1.-CValores medios de las variables explicativas utilizadas en el análisis

\begin{tabular}{|c|c|c|c|c|c|c|}
\hline \multirow[b]{2}{*}{ Variable } & \multicolumn{2}{|c|}{ Sin formación } & \multicolumn{2}{|c|}{ No financiada } & \multicolumn{2}{|c|}{ Financiada } \\
\hline & Hombres & Mujeres & Hombres & Mujeres & Hombres & Mujeres \\
\hline \multicolumn{7}{|l|}{ Nivel educativo } \\
\hline $1^{\mathrm{er}}$ nivel secundaria & 0,29 & 0,26 & 0,18 & 0,16 & 0,16 & 0,09 \\
\hline FP1 & 0,08 & 0,09 & 0,16 & 0,13 & 0,08 & 0,06 \\
\hline $2^{\circ}$ nivel secundaria & 0,11 & 0,13 & 0,12 & 0,16 & 0,19 & 0,19 \\
\hline FP2 & 0,08 & 0,08 & 0,25 & 0,18 & 0,14 & 0,09 \\
\hline Diplomado & 0,05 & 0,12 & 0,10 & 0,16 & 0,17 & 0,27 \\
\hline Licenciado & 0,08 & 0,12 & 0,13 & 0,19 & 0,18 & 0,27 \\
\hline Vive en pareja & 0,70 & 0,58 & 0,42 & 0,37 & 0,81 & 0,67 \\
\hline Experiencia & 20,52 & 15,88 & 10,86 & 8,35 & 19,91 & 16,98 \\
\hline Temporal & 0,37 & 0,41 & 0,55 & 0,61 & 0,12 & 0,21 \\
\hline \multicolumn{7}{|l|}{ Antigüedad } \\
\hline Menos de 3 años & 0,42 & 0,46 & 0,68 & 0,73 & 0,18 & 0,25 \\
\hline Temporal y menos de tres años & 0,30 & 0,34 & 0,52 & 0,57 & 0,10 & 0,16 \\
\hline \multicolumn{7}{|l|}{ Ocupación } \\
\hline Directivo & 0,03 & 0,01 & 0,02 & 0,01 & 0,08 & 0,03 \\
\hline Profesional & 0,07 & 0,15 & 0,14 & 0,21 & 0,20 & 0,40 \\
\hline Técnico & 0,09 & 0,10 & 0,13 & 0,13 & 0,19 & 0,17 \\
\hline Oficinistas & 0,07 & 0,18 & 0,09 & 0,25 & 0,15 & 0,21 \\
\hline Dependientes, seguridad & 0,11 & 0,23 & 0,10 & 0,26 & 0,10 & 0,12 \\
\hline Cualificado agricultura & 0,02 & 0,00 & 0,01 & 0,01 & 0,01 & 0,00 \\
\hline Cualificado industria & 0,29 & 0,07 & 0,22 & 0,03 & 0,16 & 0,01 \\
\hline Operadores & 0,15 & 0,04 & 0,12 & 0,02 & 0,07 & 0,02 \\
\hline Fuerzas armadas & 0,01 & 0,00 & 0,01 & 0,00 & 0,02 & 0,00 \\
\hline \multicolumn{7}{|l|}{ Sector de actividad } \\
\hline Agricultura y pesca & 0,05 & 0,02 & 0,04 & 0,01 & 0,01 & 0,00 \\
\hline Construcción & 0,18 & 0,01 & 0,11 & 0,02 & 0,04 & 0,01 \\
\hline Banca y servicios a empresas & 0,07 & 0,12 & 0,12 & 0,13 & 0,19 & 0,15 \\
\hline Comercio & 0,13 & 0,17 & 0,14 & 0,16 & 0,10 & 0,07 \\
\hline Transporte & 0,07 & 0,03 & 0,04 & 0,03 & 0,07 & 0,03 \\
\hline
\end{tabular}



FINANCIADA POR EMPRESAS Y TRABAJADORES

TABLA A1 (cont.)._-Valores medios de las variables explicativas utilizadas en el análisis

\begin{tabular}{|l|c|c|c|c|c|c|}
\hline & \multicolumn{2}{|c|}{ Sin formación } & \multicolumn{2}{c|}{ No financiada } & \multicolumn{2}{c|}{ Financiada } \\
\hline \multicolumn{1}{|c|}{ Variable } & Hombres & Mujeres & Hombres & Mujeres & Hombres & Mujeres \\
\hline Hostelería & 0,05 & 0,08 & 0,04 & 0,08 & 0,02 & 0,02 \\
\hline Administración pública & 0,06 & 0,06 & 0,08 & 0,05 & 0,14 & 0,14 \\
\hline Educación & 0,03 & 0,10 & 0,05 & 0,14 & 0,09 & 0,27 \\
\hline Sanidad & 0,02 & 0,11 & 0,05 & 0,17 & 0,04 & 0,18 \\
\hline Otras & 0,04 & 0,12 & 0,04 & 0,09 & 0,02 & 0,04 \\
\hline Tiempo parcial & 0,02 & 0,15 & 0,08 & 0,16 & 0,01 & 0,05 \\
\hline Tamaño del establecimiento & & & & & & \\
\hline Menos de 20 trabajadores & 0,46 & 0,48 & 0,43 & 0,52 & 0,26 & 0,24 \\
\hline 20 a 99 trabajadores & 0,26 & 0,26 & 0,30 & 0,27 & 0,24 & 0,33 \\
\hline Desajuste educativo & & & & & & \\
\hline Sobreeducado 1 & 0,29 & 0,34 & 0,47 & 0,47 & 0,49 & 0,51 \\
\hline Sobreeducado 2 & 0,26 & 0,24 & 0,27 & 0,23 & 0,16 & 0,13 \\
\hline Infraeducado & 0,27 & 0,20 & 0,07 & 0,06 & 0,10 & 0,03 \\
\hline Año & & & & & & \\
\hline 1996 & 0,15 & 0,14 & 0,14 & 0,15 & 0,13 & 0,11 \\
\hline 1997 & 0,17 & 0,17 & 0,18 & 0,20 & 0,23 & 0,21 \\
\hline 1998 & 0,17 & 0,18 & 0,18 & 0,21 & 0,21 & 0,20 \\
\hline 1999 & 0,17 & 0,19 & 0,18 & 0,16 & 0,17 & 0,19 \\
\hline 2000 & 0,17 & 0,19 & 0,16 & 0,17 & 0,16 & 0,20 \\
\hline Tasa de paro regional & 18,82 & 17,97 & 18,39 & 18,04 & 18,13 & 17,82 \\
\hline Número de observaciones & 12.504 & 6,238 & 790 & 778 & 1.281 & 777 \\
\hline
\end{tabular}

Nota: Se omite la categoría de referencia.

TABLA A2.-Estimaciones de los modelos tobit de efectos aleatorios para la duración de la formación no financiada

\begin{tabular}{|c|c|c|c|c|c|c|}
\hline \multirow[b]{2}{*}{ Variable } & \multicolumn{3}{|c|}{ Hombres } & \multicolumn{3}{|c|}{ Mujeres } \\
\hline & & & Err. Est. & & & Err. Est. \\
\hline \multicolumn{7}{|l|}{ Nivel educativo } \\
\hline $1^{\mathrm{er}}$ nivel secundaria & 71,3325 & $* *$ & 28,5556 & 169,8823 & $* * *$ & 38,9756 \\
\hline FP1 & 261,2962 & $* * *$ & 32,5286 & 294,6858 & $* * *$ & 42,9972 \\
\hline $2^{\circ}$ nivel secundaria & 155,2833 & $* * *$ & 33,9483 & 251,9878 & $* * *$ & 42,7811 \\
\hline
\end{tabular}


TABLA A2 (cont.)._-Estimaciones de los modelos tobit de efectos aleatorios para la duración de la formación no financiada

\begin{tabular}{|c|c|c|c|c|c|c|}
\hline \multirow[b]{2}{*}{ Variable } & \multicolumn{3}{|c|}{ Hombres } & \multicolumn{3}{|c|}{ Mujeres } \\
\hline & \multicolumn{2}{|c|}{ Coef } & \multirow{2}{*}{$\frac{\text { Err. Est. }}{32,6070}$} & \multicolumn{2}{|c|}{ Coef } & \multirow{2}{*}{$\frac{\text { Err. Est. }}{44,9980}$} \\
\hline FP2 & 317,2485 & $* * *$ & & 347,1103 & $* * *$ & \\
\hline Diplomado & 186,5615 & $* * *$ & 42,3854 & 212,7624 & $* * *$ & 47,0790 \\
\hline Licenciado & 137,6784 & *** & 43,1062 & 231,1437 & $* * *$ & 48,6871 \\
\hline Vive en pareja & $-42,7007$ & $* *$ & 19,1040 & $-88,0894$ & $* * *$ & 17,2510 \\
\hline Experiencia & $-7,8915$ & $* * *$ & 0,9965 & $-6,8462$ & $* * *$ & 1,0550 \\
\hline Temporal & $-71,5924$ & $*$ & 42,4228 & 11,3919 & & 40,8507 \\
\hline \multicolumn{7}{|l|}{ Antigüedad } \\
\hline Menos de 3 años & 70,8303 & $* * *$ & 24,5111 & 117,1093 & $* * *$ & 26,3453 \\
\hline Temporal y menos de tres años & 169,0721 & $* * *$ & 47,4215 & 57,4268 & & 46,5767 \\
\hline \multicolumn{7}{|l|}{ Ocupación } \\
\hline Directivo & $-6,3405$ & & 54,7186 & 149,7589 & $*$ & 86,9092 \\
\hline Profesional & 42,9236 & & 42,8823 & 85,9050 & $*$ & 44,2645 \\
\hline Técnico & 39,6915 & & 32,7893 & 99,7978 & $* * *$ & 38,4487 \\
\hline Oficinistas & 56,1431 & & 35,3561 & 92,8722 & $* * *$ & 34,1595 \\
\hline Dependientes, seguridad & $-60,7035$ & $*$ & 34,3822 & 94,1397 & $* * *$ & 31,8831 \\
\hline Cualificado agricultura & $-49,2611$ & & 69,6092 & 344,6308 & $* * *$ & 128,1168 \\
\hline Cualificado industria & $-1,1205$ & & 26,4549 & 88,9506 & $*$ & 50,6050 \\
\hline Operadores & 15,9816 & & 30,6900 & 53,9555 & & 61,5137 \\
\hline Fuerzas armadas & $-59,3841$ & & 80,4930 & 271,1182 & $*$ & 151,9914 \\
\hline \multicolumn{7}{|l|}{ Sector de actividad } \\
\hline Agricultura y pesca & 41,8794 & & 46,6519 & 20,3694 & & 84,3561 \\
\hline Construcción & $-53,2887$ & $* *$ & 26,7359 & 96,7617 & & 64,6049 \\
\hline Banca y servicios a empresas & 42,7358 & & 29,8552 & 72,7361 & $*$ & 37,2299 \\
\hline Comercio & 23,6349 & & 26,8358 & $-4,3233$ & & 36,2898 \\
\hline Transporte & $-110,9406$ & $* * *$ & 38,3969 & 73,9078 & & 52,7152 \\
\hline Hostelería & $-2,3966$ & & 43,6905 & 66,9897 & & 43,2750 \\
\hline Administración pública & 58,5304 & $*$ & 34,8473 & 40,4405 & & 46,2942 \\
\hline Educación & 7,5788 & & 46,0021 & 133,4909 & $* * *$ & 42,4482 \\
\hline Sanidad & 133,1546 & $* * *$ & 44,2300 & 139,0006 & $* * *$ & 38,6361 \\
\hline Otras & 39,9393 & & 40,6146 & 64,7565 & & 40,4106 \\
\hline Tiempo parcial & 176,1241 & $* * *$ & 35,7545 & 24,7345 & & 22,5397 \\
\hline
\end{tabular}



FINANCIADA POR EMPRESAS Y TRABAJADORES

TABLA A2 (cont.)._-Estimaciones de los modelos tobit de efectos aleatorios para la duración de la formación no financiada

\begin{tabular}{|c|c|c|c|c|c|c|}
\hline \multirow[b]{2}{*}{ Variable } & \multicolumn{3}{|c|}{ Hombres } & \multicolumn{3}{|c|}{ Mujeres } \\
\hline & \multicolumn{2}{|c|}{ Coef } & Err. Est. & \multicolumn{2}{|c|}{ Coef } & Err. Est. \\
\hline \multicolumn{7}{|l|}{ Tamaño del establecimiento } \\
\hline Menos de 20 trabajadores & $-19,2726$ & & 19,8993 & 46,8179 & $* *$ & 21,7755 \\
\hline 20 a 99 trabajadores & 29,1507 & & 20,5443 & 48,5731 & $* *$ & 23,4566 \\
\hline \multicolumn{7}{|l|}{ Desajuste educativo } \\
\hline Sobreeducado 1 & 43,4545 & $* *$ & 20,9111 & 14,1591 & & 20,9583 \\
\hline Sobreeducado 2 & $-18,7586$ & & 23,4467 & 4,4390 & & 26,1269 \\
\hline Infraeducado & $-157,5953$ & $* * *$ & 29,8659 & $-99,7940$ & $* * *$ & 33,5365 \\
\hline \multicolumn{7}{|l|}{ Año } \\
\hline 1996 & $-3,6168$ & & 27,4840 & 38,7168 & & 30,5966 \\
\hline 1997 & 10,0210 & & 26,3586 & 83,2909 & $* * *$ & 29,3593 \\
\hline 1998 & $-9,5394$ & & 27,0929 & 34,4729 & & 29,9416 \\
\hline 1999 & $-24,1578$ & & 28,4958 & $-32,1258$ & & 32,4343 \\
\hline 2000 & $-47,1570$ & & 30,3341 & $-9,0927$ & & 33,3862 \\
\hline Tasa de paro regional & $-2,2853$ & & 1,6820 & $-2,0133$ & & 1,8832 \\
\hline Constante & $-626,2029$ & $* * *$ & 64,5989 & $-804,7467$ & $* * *$ & 80,4160 \\
\hline$\sigma_{u}$ & 28,7177 & & & 38,9524 & & \\
\hline$\sigma_{e}$ & 389,2966 & & & 368,5268 & & \\
\hline$\rho$ & 0,0054 & & & 0,0110 & & \\
\hline$\chi^{2}$ & 5,00 & & & 9,29 & & \\
\hline $\mathrm{N}$ & 4566 & & & 2743 & & \\
\hline NT & 13270 & & & 6988 & & \\
\hline Obs. no censuradas & 766 & & & 751 & & \\
\hline Obs. censuradas (izquierda) & 12504 & & & 6237 & & \\
\hline
\end{tabular}

Notas: El individuo de referencia es un trabajador con estudios primarios o sin estudios, con contrato indefinido y 3 o más años de antigüedad en la empresa, que realiza un empleo no cualificado en el sector industrial a tiempo completo en un establecimiento de 100 o más empleados, sin desajuste educativo y es entrevistado en 1995.

$* * *$ Coeficiente significativo al $1 \%, * *$ al $5 \%, *$ al $10 \%$. 
TABLA A3.-Estimaciones de los modelos tobit de efectos aleatorios para la duración de la formación financiada

\begin{tabular}{|c|c|c|c|c|c|c|}
\hline \multirow[b]{2}{*}{ Variable } & \multicolumn{3}{|c|}{ Hombres } & \multicolumn{3}{|c|}{ Mujeres } \\
\hline & \multicolumn{2}{|c|}{ Coef } & Err. Est. & \multicolumn{2}{|c|}{ Coef } & Err. Est. \\
\hline \multicolumn{7}{|l|}{ Nivel educativo } \\
\hline $1^{\mathrm{er}}$ nivel secundaria & 36,2432 & $* * *$ & 11,2590 & 73,3812 & $* * *$ & 20,3907 \\
\hline FP1 & 67,8370 & $* * *$ & 14,6256 & 77,2554 & $* * *$ & 23,3559 \\
\hline $2^{\circ}$ nivel secundaria & 69,5223 & $* * *$ & 13,3700 & 131,1516 & $* * *$ & 21,6776 \\
\hline FP2 & 98,5454 & $* * *$ & 13,9562 & 119,3071 & $* * *$ & 23,8184 \\
\hline Diplomado & 99,6217 & $* * *$ & 16,0708 & 128,5491 & $* * *$ & 23,2435 \\
\hline Licenciado & 60,9975 & $* * *$ & 16,4848 & 132,4071 & $* * *$ & 24,1171 \\
\hline Vive en pareja & 30,5613 & $* * *$ & 9,2034 & 20,3255 & $* *$ & 8,0087 \\
\hline Experiencia & $-1,4921$ & $* * *$ & 0,3786 & 0,1528 & & 0,4356 \\
\hline Temporal & $-60,3059$ & $* * *$ & 16,2736 & 1,2669 & & 16,7431 \\
\hline \multicolumn{7}{|l|}{ Antigüedad } \\
\hline Menos de 3 años & $-40,2437$ & $* * *$ & 10,6180 & $-36,1565$ & $* * *$ & 13,4701 \\
\hline Temporal y menos de tres años & 43,1939 & $* *$ & 19,7202 & 2,4715 & & 21,8171 \\
\hline \multicolumn{7}{|l|}{ Ocupación } \\
\hline Directivo & 94,4609 & $* * *$ & 18,9502 & 137,9277 & $* * *$ & 32,7509 \\
\hline Profesional & 57,1525 & $* * *$ & 18,2609 & 85,8696 & $* * *$ & 22,1754 \\
\hline Técnico & 60,6962 & $* * *$ & 15,4408 & 84,6274 & $* * *$ & 20,2968 \\
\hline Oficinistas & 70,5061 & $* * *$ & 16,1223 & 64,4840 & *** & 19,1130 \\
\hline Dependientes, seguridad & 47,2947 & $* * *$ & 16,2428 & 74,9333 & $* * *$ & 19,2588 \\
\hline Cualificado agricultura & $-6,4646$ & & 38,6491 & 125,2851 & & 88,6539 \\
\hline Cualificado industria & 22,5572 & & 14,3334 & $-32,6691$ & & 33,0666 \\
\hline Operadores & 8,1769 & & 15,9355 & 29,7222 & & 30,7563 \\
\hline Fuerzas armadas & 51,4739 & $*$ & 29,3787 & 86,3393 & & 100,1922 \\
\hline \multicolumn{7}{|l|}{ Sector de actividad } \\
\hline Agricultura y pesca & $-22,0839$ & & 27,5460 & $-24,9520$ & & 55,2365 \\
\hline Construcción & $-35,9606$ & $* * *$ & 13,7957 & $-5,6779$ & & 36,2085 \\
\hline Banca y servicios a empresas & 42,3576 & $* * *$ & 12,1008 & 18,6453 & & 16,9398 \\
\hline Comercio & $-8,8667$ & & 12,2271 & $-41,8095$ & $* *$ & 18,5602 \\
\hline Transporte & $-3,2465$ & & 14,0500 & $-38,3801$ & & 25,2335 \\
\hline Hostelería & $-47,0794$ & $* *$ & 22,1672 & $-25,8198$ & & 24,9662 \\
\hline Administración pública & 20,4699 & & 13,3245 & 19,4887 & & 18,0903 \\
\hline
\end{tabular}


TABLA A3 (cont.).-Estimaciones de los modelos tobit de efectos aleatorios para la duración de la formación financiada

\begin{tabular}{|c|c|c|c|c|c|c|}
\hline \multirow[b]{2}{*}{ Variable } & \multicolumn{3}{|c|}{ Hombres } & \multicolumn{3}{|c|}{ Mujeres } \\
\hline & \multicolumn{2}{|c|}{ Coef } & \multirow{2}{*}{$\begin{array}{c}\text { Err. Est. } \\
17,6173\end{array}$} & \multicolumn{2}{|c|}{ Coef } & \multirow{2}{*}{$\begin{aligned} & \text { Err. Est. } \\
& 18,0082\end{aligned}$} \\
\hline Educación & 59,6760 & $* * *$ & & 48,2140 & $* * *$ & \\
\hline Sanidad & 23,4455 & & 19,0080 & 1,4300 & & 17,5641 \\
\hline Otras & $-10,3916$ & & 19,5431 & $-24,1959$ & & 21,7738 \\
\hline Tiempo parcial & 13,8248 & & 23,4912 & $-32,8467$ & $* *$ & 14,2168 \\
\hline \multicolumn{7}{|l|}{ Tamaño del establecimiento } \\
\hline Menos de 20 trabajadores & $-49,9279$ & $* * *$ & 7,9664 & $-61,0728$ & $* * *$ & 10,0240 \\
\hline 20 a 99 trabajadores & $-27,9937$ & $* * *$ & 7,9292 & $-21,9975$ & $* *$ & 9,6626 \\
\hline \multicolumn{7}{|l|}{ Desajuste educativo } \\
\hline Sobreeducado 1 & 10,8296 & & 7,3962 & 11,7899 & & 8,9007 \\
\hline Sobreeducado 2 & $-17,7003$ & $*$ & 9,3944 & 2,8795 & & 12,6855 \\
\hline Infraeducado & $-35,2958$ & $* * *$ & 10,4589 & $-60,5397$ & $* * *$ & 18,0189 \\
\hline \multicolumn{7}{|l|}{ Año } \\
\hline 1996 & 25,2701 & $* *$ & 10,6964 & 31,7008 & $* *$ & 15,9015 \\
\hline 1997 & 47,8121 & $* * *$ & 10,1577 & 36,7753 & $* *$ & 14,8126 \\
\hline 1998 & 41,5908 & $* * *$ & 10,6337 & 28,8995 & * & 15,1275 \\
\hline 1999 & 16,3310 & & 11,6658 & 16,6036 & & 16,0340 \\
\hline 2000 & 13,4519 & & 12,4832 & 32,3028 & * & 16,5993 \\
\hline Tasa de paro regional & $-0,5510$ & & 0,7780 & 1,4593 & * & 0,8777 \\
\hline Constante & $-301,2353$ & $* * *$ & 28,4748 & $-409,1562$ & $* * *$ & 38,8842 \\
\hline$\sigma_{u}$ & 104,9510 & & & 13,1742 & & \\
\hline$\sigma_{e}$ & 142,2527 & & & 170,2096 & & \\
\hline$\rho$ & 0,3525 & & & 0,0060 & & \\
\hline$\chi^{2}$ & 298,73 & & & 4,55 & & \\
\hline $\mathrm{N}$ & 4521 & & & 2651 & & \\
\hline NT & 13755 & & & 7000 & & \\
\hline Obs. no censuradas & 1251 & & & 763 & & \\
\hline Obs. censuradas (izquierda) & 12504 & & & 6237 & & \\
\hline
\end{tabular}

Notas: El individuo de referencia es un trabajador con estudios primarios o sin estudios, con contrato indefinido y 3 o más años de antigüiedad en la empresa, que realiza un empleo no cualificado en el sector industrial a tiempo completo en un establecimiento de 100 o más empleados, sin desajuste educativo y es entrevistado en 1995.

$* * *$ Coeficiente significativo al $1 \%, * *$ al $5 \%, *$ al $10 \%$. 